\title{
FILLINGS AND FITTINGS OF UNIT COTANGENT BUNDLES OF ODD-DIMENSIONAL SPHERES
}

\author{
MYEONGGI KWON AND KAI ZEHMISCH
}

\begin{abstract}
We introduce the concept of fittings to symplectic fillings of the unit cotangent bundle of odd-dimensional spheres. Assuming symplectic asphericity we show that all fittings are diffeomorphic to the respective unit co-disc bundle.
\end{abstract}

\section{INTRODUCTION}

There is a general question in symplectic geometry: How much does the boundary of a compact symplectic manifold know about its interior? When the boundary is of contact type, the question becomes meaningful. In fact, this question is of high current interest in dimension 4. But much less is known about the topology of the fillings in higher dimensions. By results of Eliashberg-Floer-McDuff, see [6. Theorem 1.5], and Barth-Geiges-Zehmisch [2] the diffeomorphism type of symplectically aspherical fillings is uniquely determined for many subcritically Stein fillable contact manifolds. But almost nothing is known in the critical case so far. This paper is about symplectically aspherical fillings of the unit cotangent bundle of odd-dimensional spheres. These are never subcritically Stein by [2, Theorem 1.2] because the unit cotangent bundle is of critical Stein type. For basic notions the reader is referred to Geiges' book 4 .

For a given natural number $d$ we consider the cotangent bundle $T^{*} S^{2 d+1}$ of the round $(2 d+1)$-dimensional sphere. Like every cotangent bundle, $T^{*} S^{2 d+1}$ admits the Liouville canonical 1-form $\lambda$, which in local coordinates is given by $\mathbf{p} \mathrm{d} \mathbf{q}$. The induced contact structure on the unit cotangent bundle

$$
M=S T^{*} S^{2 d+1}
$$

is denoted by $\xi$. The resulting contact manifold $(M, \xi)$ admits a natural symplectic filling $\left(W_{\mathrm{st}}, \omega_{\mathrm{st}}\right)$ given by the unit disc bundle $\left(D T^{*} S^{2 d+1}, \mathrm{~d} \lambda\right)$. We call $\left(W_{\mathrm{st}}, \omega_{\mathrm{st}}\right)$ the standard filling.

We would like to find natural conditions under which a symplectic filling $(W, \omega)$ of $(M, \xi)$ is diffeomorphic to $W_{\text {st }}$. Observe that a blow up of $(W, \omega)$ at any interior point results in a new symplectic filling of $(M, \xi)$, which cannot be diffeomorphic to $W_{\text {st. }}$. The reason is that diffeomorphically the blow up is a connected sum with $\mathbb{C} P^{4 d+2}$ with the opposite orientation and therefore contains a homologically nontrivial symplectic 2 -sphere. In order to exclude this ambiguity we require all fillings

Date: 02.04 .2019$.

2010 Mathematics Subject Classification. 57R17; 32Q65, 53D35, 57R80.

This research is part of a project in the SFB/TRR 191 Symplectic Structures in Geometry, Algebra and Dynamics, funded by the DFG. 
to be symplectically aspherical, that is we require the symplectic form to be zero evaluated on all spherical homology 2-classes.

Unfortunately, symplectic asphericity seems not to be rigid enough to determine the diffeomorphism type of symplectic fillings of the unit cotangent bundle $(M, \xi)$ of $S^{2 d+1}$. That's why we want to search for an additional condition. In dimension 4 holomorphic curves as such are often self-dual in a symplectic way and in terms of Poincaré duality. This is crucial for uniqueness of symplectic fillings in dimension 4 . Therefore, we are looking for symplectic hypersurfaces inside a given filling $(W, \omega)$ that behave nicely w.r.t. the boundary of $(W, \omega)$ and that remember a sufficiently large amount of symplectic informations of $\left(W_{\mathrm{st}}, \omega_{\mathrm{st}}\right)$.

In the given situation this means the following: The standard filling $\left(W_{\mathrm{st}}, \omega_{\mathrm{st}}\right)$ itself appears as a Weinstein neighbourhood of the graph of the anti-Hopf map inside $\mathbb{C}^{d+1} \times \mathbb{C} P^{d}$, see Section 3.1, and embeds into the obvious partial compactification $\mathbb{C} P^{1} \times \mathbb{C}^{d} \times \mathbb{C} P^{d}$. The intersection of $W_{\text {st }}$ with $\mathbb{C} P^{1} \times \mathbb{C}^{d} \times \mathbb{C} P^{d-1}$ defines a complex hypersurface in $\left(W_{\text {st }}, \omega_{\text {st }}\right)$ relative to the boundary. This complex hypersurface inherits the same homologically trivial intersection behaviour as holomorphic spheres $\mathbb{C} P^{1} \times \mathrm{pt} \times \mathrm{pt}$ and complex hypersurfaces $\mathbb{C} P^{1} \times \mathbb{C}^{d} \times \mathbb{C} P^{d-1}$ have inside $\mathbb{C} P^{1} \times \mathbb{C}^{d} \times \mathbb{C} P^{d}$. We call a filling that admits such a complex hypersurface a fitting, see Section 4.1. As it will turn out fittings are the right concept to ensure uniqueness:

Theorem 1.1. If $(W, \omega)$ is a symplectically aspherical fitting of $(M, \xi)$, then $W$ and $W_{\mathrm{st}}$ are diffeomorphic.

\section{From homology GROUPS TO DIFFEOMORPHISM TYPE}

In Section 3 we will verify the assumptions of the following proposition for fittings. The argumentation will be similar to earlier work which can be found in 2, Section 5] and [3, Section 4].

Proposition 2.1. Let $(W, \omega)$ be a symplectically aspherical filling of $(M, \xi)$. If $W$ is simply connected and has the homology type of $W_{\text {st }}$, i.e. $H_{k} W=H_{k} W_{\text {st }}$ for all $k$, then $W$ is diffeomorphic to $W_{\mathrm{st}}$. In other words, the diffeomorphism type of $W$ is unique in this case.

This section is intended to provide evidence for this statement.

2.1. A cobordism. We consider the cotangent disc bundle $D_{R} T^{*} S^{2 d+1}$ of radius $R>1$ so that the unit cotangent disc bundle $W_{\mathrm{st}}=D T^{*} S^{2 d+1}$ appears as a subset of $D_{R} T^{*} S^{2 d+1}$. Via gluing of smooth manifolds with boundary we define

$$
W_{1}:=\left(D_{R} T^{*} S^{2 d+1} \backslash \operatorname{Int} W_{\mathrm{st}}\right) \cup_{M} W
$$

by replacing $W_{\text {st }}$ with $W$ inside $D_{R} T^{*} S^{2 d+1}$. For sufficiently large radius $R$ the spherical shell bundle $\left(D_{R} T^{*} S^{2 d+1} \backslash \operatorname{Int} W_{\text {st }}\right)$ contains a copy $W_{0}$ of $W_{\text {st }}$. This copy of $W_{\text {st }}$ is obtained by first taking a section $s$ of the sphere bundle $S T^{*} S^{2 d+1} \rightarrow S^{2 d+1}$ and then shifting $D T^{*} S^{2 d+1}$ suitably inside $T^{*} S^{2 d+1}$ in the direction of $s$. This shifted copy of $W_{\text {st }}$ is also considered to be contained in $W_{1}$. We define a cobordism $X$ between $M_{0}:=\partial W_{0}$ and $M_{1}:=\partial W_{1}$ by

$$
X:=W_{1} \backslash \operatorname{Int} W_{0} .
$$


Remark 2.2. The construction of the cobordism $X$ heavily relies on the existence of a nowhere vanishing vector field on $S^{2 d+1}$. This is the first of two places in our argument that requires the spheres $S^{2 d+1}$ to have odd dimensions. The second one is the use of the Hopf fibration in Section 3.1 .

2.2. Applying the $h$-cobordism theorem. Under the assumptions of Proposition 2.1 the cobordism $X$ is an $h$-cobordism: The boundary components $M_{0}$ and $M_{1}$ are both $S^{2 d}$-bundles over $S^{2 d+1}, d \geq 1$, and, hence, simply connected thanks to the corresponding homotopy sequence. The cobordism $X$ itself is simply connected too. Indeed, $W_{1} \simeq W$ is simply connected by assumption. The contraction $S^{2 d+1} \simeq W_{\text {st }}$ induces a contraction $S \simeq W_{0}$ of $W_{0}$ onto the shifted copy $S$ of $S^{2 d+1}$ under the shift that we used in the construction of $X$ in Section 2.1. This contraction defines a contraction of $W_{1} \backslash S$ onto Int $X$. Simply connectedness of $X$ now follows with the simply connectedness of $W_{1}$ together with a general position argument that uses $2+(2 d+1)<4 d+2=\operatorname{dim} W_{1}$. It follows that the inclusions $M_{0} \hookrightarrow X$ and $M_{1} \hookrightarrow X$ of the boundaries induce isomorphisms of the respective fundamental groups.

Lemma 2.3. $H_{*}\left(X, M_{0}\right)=0$.

Proof of Proposition 2.1. With Lemma 2.3. Poincaré-Lefschetz duality and the universal coefficient theorem imply $H_{*}\left(X, M_{1}\right)=0$. A combination of Hurewicz' and Whitehead's theorem implies that the inclusions $M_{0} \hookrightarrow X$ and $M_{1} \hookrightarrow X$ are strong deformation retracts. This uses our preceding considerations on the simply connectedness of $M_{0}, M_{1}$, and $X$. In other words $\left\{M_{0}, X, M_{1}\right\}$ is a simply connected $h$-cobordism of dimension $4 d+2 \geq 6$.

The $h$-cobordism theorem implies that $X$ is diffeomorphic to $[0,1] \times M_{0}$ via a diffeomorphism that restricts to the identity on $0 \times M_{0}$, see $\left[8\right.$. Gluing back $W_{0}$ to $X$ and extending the diffeomorphism suitably we obtain that $W_{1}$ is diffeomorphic to a collar extension of $W_{0}$. It follows that $W$ and $W_{\text {st }}$ are diffeomorphic.

2.3. Killing relative homology. It remains to prove Lemma2.3. By the excision axiom this lemma is equivalent to $H_{*}\left(W_{1}, W_{0}\right)=0$.

To show homology vanishing of the pair $\left(W_{1}, W_{0}\right)$ we observe that $S^{2 d+1} \simeq W_{0}$, so that, invoking our assumptions, $H_{k} S^{2 d+1}=H_{k} W_{0}=H_{k} W_{1}$ for all $k \in \mathbb{Z}$. The long exact sequence of $\left(W_{1}, W_{0}\right)$ implies $H_{k}\left(W_{1}, W_{0}\right)=0$ for all $k \neq 2 d+1,2 d+2$ and exactness of

$$
0 \rightarrow H_{2 d+2}\left(W_{1}, W_{0}\right) \rightarrow H_{2 d+1} W_{0} \rightarrow H_{2 d+1} W_{1} \rightarrow H_{2 d+1}\left(W_{1}, W_{0}\right) \rightarrow 0 .
$$

Therefore, in order to prove Proposition 2.1 it suffices to show that the map $\iota_{*}: H_{2 d+1} W_{0} \rightarrow H_{2 d+1} W_{1}$ induced by the inclusion $W_{0} \subset W_{1}$ is invertible.

We will use the cobordism diagram below. To explain the cobordism diagram we consider the shift map that brings $W_{\mathrm{st}}$ to $W_{0}$ in the construction we gave in Section 2.1. The image of the section $s$, which is contained in $\partial W_{\text {st }}$, is mapped to $S_{0} \subset M_{0}$ under the shift map. Also, with the construction of $X$ we find a diffeomorphism $M_{0} \rightarrow M_{1}$ that maps $S_{0}$ onto the section $S_{1}$, say, such that $S_{0}$ is isotopic to $S_{1}$ inside $W_{1}$. This isotopy extends to an isotopy of $W_{0}$ inside $W_{1}$ resulting in the following homotopy commutative diagram: 


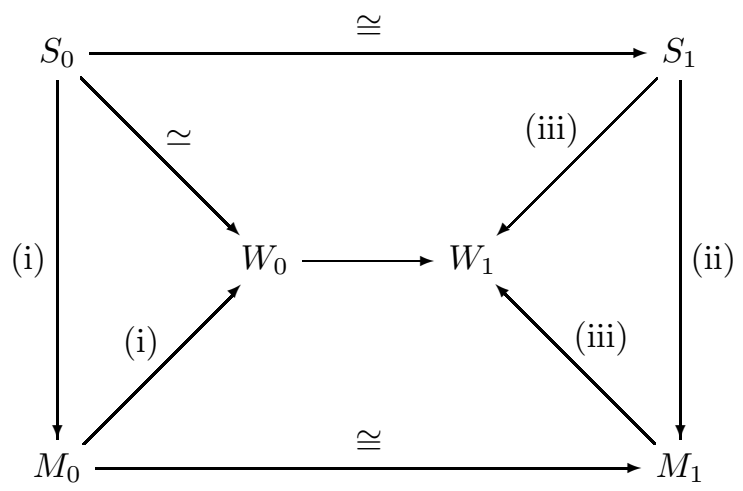

We would like to understand the diagram in homology of degree $2 d+1$. Starting with the left triangle we see that the inclusion of the $(2 d+1)$-sphere $S_{0}$ into $W_{0}$ is a homotopy equivalence. Using the Gysin sequence of the Euler class zero bundle $S T^{*} S^{2 d+1} \rightarrow S^{2 d+1}$ and the universal coefficient theorem we see that the homology of $M_{0}$ in degree $2 d+1$ equals $\mathbb{Z}$. Therefore, all morphisms of the left triangle in degree $2 d+1$ are multiplication by \pm 1 , i.e. the maps (i) are $H_{2 d+1}$-isomorphisms.

Using the outer square we see that the map (ii) is a $H_{2 d+1}$-isomorphism too. We claim that the maps (iii) are $H_{2 d+1}$-isomorphisms. Our assumptions on the homology type of $W$ translate into $H_{*} W_{1}=H_{*} W_{0}$. Poincaré-Lefschetz duality and the universal coefficient theorem imply $H_{2 d+2}\left(W_{1}, M_{1}\right)=0$ and $H_{2 d+1}\left(W_{1}, M_{1}\right)=$ $\mathbb{Z}$. Therefore, the long exact sequence for the pair $\left(W_{1}, M_{1}\right)$ implies exactness of

$$
0 \rightarrow H_{2 d+1} M_{1} \rightarrow H_{2 d+1} W_{1} \rightarrow \mathbb{Z} \rightarrow \mathbb{Z} \rightarrow 0,
$$

where we used that $H_{2 d} M_{1}=\mathbb{Z}$ by the Gysin sequence of the Euler class zero bundle $S T^{*} S^{2 d+1} \rightarrow S^{2 d+1}$ and the universal coefficient theorem. As any surjection $\mathbb{Z} \rightarrow \mathbb{Z}$ is necessarily invertible, $H_{2 d+1} M_{1} \rightarrow H_{2 d+1} W_{1}$ is indeed an isomorphism. Therefore, the maps labeled (iii) are isomorphisms in $H_{2 d+1}$ as claimed.

Therefore, all morphisms in the cobordism diagram are $H_{2 d+1}$-isomorphisms. In particular, $\iota_{*}: H_{2 d+1} W_{0} \rightarrow H_{2 d+1} W_{1}$ is invertible. This completes the proof of Lemma 2.3 .

\section{HolOMORPHic FILLING VIA CAP CONSTRUCTIONS}

Cap constructions were used in [5, 9] to verify instances of the Weinstein conjecture, and in 2] for classifications of subcritical fillings. In the critical case too, symplectic caps are essential.

3.1. The anti-Hopf map and capping. The unit sphere $S^{2 d+1} \subset \mathbb{C}^{d+1}, d \geq 1$, fibres over $\mathbb{C} P^{d}$ via the Hopf map $S^{2 d+1} \rightarrow \mathbb{C} P^{d}, z \mapsto[z]$. As observed by AudinLalonde-Polterovich ([1]) the graph of the anti-Hopf map

$$
S^{2 d+1} \longrightarrow \mathbb{C}^{d+1} \times \mathbb{C} P^{d}, \quad z \longmapsto(z,[\bar{z}]),
$$

defines a Lagrangian embedding, which by the Weinstein neighbourhood theorem extends to a symplectic embedding of a neighbourhood of the zero section of the 
cotangent bundle $T^{*} S^{2 d+1}$. Rescaling the metric on $S^{2 d+1}$ suitably, this defines an embedding of $D T^{*} S^{2 d+1}$ into $\mathbb{C}^{d+1} \times \mathbb{C} P^{d}$, and we may assume that

$$
(M, \xi)=S T^{*} S^{2 d+1} \subset \mathbb{C}^{d+1} \times \mathbb{C} P^{d}
$$

appears as a hypersurface of contact type. The complement of the interior of $D T^{*} S^{2 d+1}$ is a so-called symplectic cap

$$
\text { Cap : }=\mathbb{C}^{d+1} \times \mathbb{C} P^{d} \backslash \operatorname{Int}\left(D T^{*} S^{2 d+1}\right)
$$

and carries the symplectic form

$$
\omega_{\mathrm{Cap}}:=\mathrm{d} x \wedge \mathrm{d} y \oplus \omega_{\mathrm{FS}} .
$$

We define a symplectic manifold

$$
(Z, \Omega):=(W, \omega) \cup_{(M, \xi)}\left(\text { Cap, } \omega_{\text {Cap }}\right)
$$

via gluing along contact type boundaries. Choose $z_{0} \in \mathbb{C}$ such that the complex hypersurface $z_{0} \times \mathbb{C}^{d} \times \mathbb{C} P^{d}$ is contained in $Z$.

Lemma 3.1. If the embedding $z_{0} \times \mathbb{C}^{d} \times \mathbb{C} P^{d} \rightarrow Z$ is $\pi_{1}$-surjective and $H_{k}(. ; \mathbb{F})$ onto for all $k \in \mathbb{Z}$ and all fields $\mathbb{F}$, then $W$ and $W_{\mathrm{st}}$ are diffeomorphic.

Proof. In view of Proposition 2.1 we need to show that $\pi_{1} W=1$ and $H_{*} W=$ $H_{*} W_{\mathrm{st}}$.

By the $\pi_{1}$-surjectivity assumption, we have $\pi_{1} Z=1$, since $\mathbb{C} P^{d}$ is simply connected. As $S^{2 d+1} \subset \mathbb{C}^{d+1} \times \mathbb{C} P^{d}$ has codimension $\geq 3$, we get that Cap is simply connected. By the Seifert-van Kampen theorem, $\pi_{1} Z$ is the free product of $\pi_{1} W$ and the trivial group, because $M$ is simply connected. It follows that $\pi_{1} W=1$.

By assumption, the induced map $H_{*}\left(\mathbb{C}^{d} ; \mathbb{F}\right) \rightarrow H_{*}(Z ; \mathbb{F})$ is surjective for all fields $\mathbb{F}$. With the universal coefficient theorem, we find $H_{k} Z=0$ for all $k \geq 2 d+1$. The Mayer-Vietoris sequence for the decomposition $Z=W \cup$ Cap along $M$ gives $H_{k} M=H_{k} W \oplus H_{k}(\mathrm{Cap})$ for all $k \geq 2 d+1$. Similarly, $H_{k} M=H_{k} W_{\mathrm{st}} \oplus H_{k}(\mathrm{Cap})$ for all $k \geq 2 d+1$, so that $H_{k} W=H_{k} W_{\text {st }}$ for all $k \geq 2 d+1$.

To prove the isomorphism $H_{k} W=H_{k} W_{\text {st }}$ for all $k \leq 2 d$, observe that by excision and Poincaré-Lefschetz duality applied to $(Z, W)$ we have $H^{k} W=H_{4 d+2-k}(Z$, Cap) for all $k \in \mathbb{Z}$. Note that $k \leq 2 d$ translates into $4 d+2-k \geq 2 d+2$ and that the long exact sequence of the pair $\left(Z\right.$, Cap) gives $H_{m}(Z$, Cap $)=H_{m-1}($ Cap $)$ for all $m \geq 2 d+2$. Hence, $H^{k} W=H_{4 d+1-k}($ Cap $)$ for all $k \leq 2 d$. Similarly, $H^{k} W_{\mathrm{st}}=H_{4 d+1-k}(\mathrm{Cap})$ for all $k \leq 2 d$ using excision of the pair $\left(\mathbb{C}^{d+1} \times \mathbb{C} P^{d}, W_{\mathrm{st}}\right)$ and Poincaré-Lefschetz duality. We conclude $H^{k} W=H^{k} W_{\text {st }}$ for all $k \leq 2 d$, which in fact holds true in homology with any field coefficients. The universal coefficient theorem implies $H_{k} W=H_{k} W_{\text {st }}$ for all $k \leq 2 d$, as the reduced homology of $S^{2 d+1}$ vanishes in all degrees $\leq 2 d$.

3.2. A cap construction. As a first step in defining a moduli space of holomorphic spheres we will partially compactify $\mathbb{C}^{d+1} \times \mathbb{C} P^{d}$. The subset

$$
B_{r}^{2}(0) \times \mathbb{C}^{d} \times \mathbb{C} P^{d}, \quad r>1,
$$

of $\mathbb{C}^{d+1} \times \mathbb{C} P^{d}$ completes symplectically to

$$
\left(\mathbb{C} P^{1} \times \mathbb{C}^{d} \times \mathbb{C} P^{d}, r^{2} \omega_{\mathrm{FS}} \oplus \mathrm{d} x \wedge \mathrm{d} y \oplus \omega_{\mathrm{FS}}\right)
$$

where $\omega_{\mathrm{FS}}$ stands for the Fubini-Study form of the respective projective space. The Lagrangian image of $S^{2 d+1}$ under the anti-Hopf map is contained in $B_{r}^{2}(0) \times \mathbb{C}^{d} \times$ 
$\mathbb{C} P^{d}$. By rescaling the round metric on $S^{2 d+1}$ as in Section $3.1,(M, \xi)=S T^{*} S^{2 d+1}$ also appears as a hypersurface of contact type in $\mathbb{C} P^{1} \times \mathbb{C}^{d} \times \mathbb{C} P^{d}$ provided with the symplectic form

$$
\hat{\omega}:=r^{2} \omega_{\mathrm{FS}} \oplus \mathrm{d} x \wedge \mathrm{d} y \oplus \omega_{\mathrm{FS}} .
$$

We equip the complement

$$
\widehat{\mathrm{Cap}}:=\mathbb{C} P^{1} \times \mathbb{C}^{d} \times \mathbb{C} P^{d} \backslash \operatorname{Int}\left(D T^{*} S^{2 d+1}\right)
$$

with the symplectic form induced by $\hat{\omega}$ and obtain a symplectic cap. $\widehat{\text { Cap }}$ contains holomorphic spheres

$$
C_{1}:=\mathbb{C} P^{1} \times \mathrm{pt} \times \mathrm{pt}, \quad C_{2}:=\mathrm{pt} \times \mathrm{pt} \times \mathbb{C} P^{1},
$$

where pt stands for a generic choice of a point in the respective factor. In the definition of $C_{2}, \mathbb{C} P^{1} \subset \mathbb{C} P^{d}$ denotes a complex line. Dual hypersurfaces are

$$
H_{1}:=\infty \times \mathbb{C}^{d} \times \mathbb{C} P^{d}, \quad H_{2}:=\mathbb{C} P^{1} \times \mathbb{C}^{d} \times \mathbb{C} P^{d-1},
$$

where $\mathbb{C} P^{d-1} \subset \mathbb{C} P^{d}$ denotes a complex hyperplane. Assuming $\mathbb{C} P^{1}$ and $\mathbb{C} P^{d-1}$ in general position in $\mathbb{C} P^{d}$ it follows for the intersection numbers that

$$
C_{i} \cdot H_{j}=\delta_{i j}
$$

for $i, j=1,2$.

3.3. A moduli space. We define a symplectic manifold

$$
(\hat{Z}, \hat{\Omega}):=(W, \omega) \cup_{(M, \xi)}(\widehat{\operatorname{Cap}}, \hat{\omega}) .
$$

It contains a subset $W_{\square}$ that by definition is the union of $W$ and all points in $\widehat{\mathrm{Cap}}$ of the form $\left(z_{1}, \mathbf{z}, w\right)$ that satisfy $\left|z_{1}\right|<R$ and $|\mathbf{z}|<R$ for some fixed $R \in(1, r)$. Let $J$ be a tame almost complex structure on $(\hat{Z}, \hat{\Omega})$ that is equal to the standard complex structure $J_{\text {st }}$ on $\hat{Z} \backslash W_{\square}$ induced by $\mathbb{C} P^{1} \times \mathbb{C}^{d} \times \mathbb{C} P^{d}$.

Lemma 3.2. For all non-constant parametrised holomorphic spheres $C=u\left(\mathbb{C} P^{1}\right)$, $u: \mathbb{C} P^{1} \rightarrow(\hat{Z}, J)$, the following hold true.

(i) If $C \subset \hat{Z} \backslash W_{\square}$, then $u=\left(u^{1}\right.$, pt, $\left.u^{3}\right)$, where pt $\in \mathbb{C}^{d}$ and $u^{1}: \mathbb{C} P^{1} \rightarrow \mathbb{C} P^{1}$, $u^{3}: \mathbb{C} P^{1} \rightarrow \mathbb{C} P^{d}$ are holomorphic.

(ii) If $C \cap\left(\hat{Z} \backslash \bar{W}_{\square}\right) \neq \emptyset$ and $C \cap H_{1}=\emptyset$, then $u=\left(\mathrm{pt}\right.$, pt, $\left.u^{3}\right)$, where $u^{3}$ : $\mathbb{C} P^{1} \rightarrow \mathbb{C} P^{d}$ is holomorphic.

Proof. (i) follows with the maximum principle. (ii) holds because the maximum principle in $\mathbb{C}$ - and in $\mathbb{C}^{d}$-direction implies that the respective projections of $C$ a posteriori are contained in the level sets of the radial distance functions. Hence, $C \subset \hat{Z} \backslash W_{\square}$ and the claim follows with (i).

The moduli space $\mathcal{M}$ consists of all holomorphic maps $u: \mathbb{C} P^{1} \rightarrow(\hat{Z}, J)$ such that $u\left(\mathbb{C} P^{1}\right)$ is homologous to $C_{1}$ and $u(z) \in z \times \mathbb{C}^{d} \times \mathbb{C} P^{d}$ for $z \in\{ \pm \varrho, \infty\}$, $\varrho \in(R, r)$.

Remark 3.3. We observe:

(i) All holomorphic spheres $u \in \mathcal{M}$ are simple because positivity of intersections implies $u \bullet H_{1}=C_{1} \cdot H_{1}=1$, see [5, 7].

(ii) The index $2 \operatorname{dim}_{\mathbb{C}}(\hat{Z})+2 c_{1}\left(C_{1}\right)-6$ of the underlying Fredholm problem equals $4 d$, because $\left.T \hat{Z}\right|_{C_{1}}=T \mathbb{C} P^{1} \oplus \underline{\mathbb{C}}^{d} \oplus \underline{\mathbb{C}}^{d}$ and, hence, $c_{1}\left(C_{1}\right)=2$. 
(iii) If $u\left(\mathbb{C} P^{1}\right) \subset \hat{Z} \backslash W_{\square}$ for $u \in \mathcal{M}$, then $u=$ (id,pt,pt). In particular $u$ is regular. Indeed, with Lemma 3.2 (i) we get $u=\left(u^{1}, \mathrm{pt}, u^{3}\right)$ for holomorphic spheres $u^{1}$ and $u^{3}$ in $\mathbb{C} P^{1}$ and $\mathbb{C} P^{d}$, resp. Because of $u \bullet H_{1}=1$ the holomorphic map $u^{1}$ is an automorphism of $\mathbb{C} P^{1}$ that fixes 3 points, i.e. $u^{1}=$ id. Therefore,

$$
r^{2} \pi=\int_{\mathbb{C} P^{1}} u^{*} \hat{\Omega}=r^{2} \pi+\int_{\mathbb{C} P^{1}}\left(u^{3}\right)^{*} \omega_{\mathrm{FS}} .
$$

The second integral is the Dirichlet energy of $u^{3}$, which has to vanish by the equation. It follows that $u^{3}$ is constant.

(iv) We choose the almost complex structure $J$ on $\hat{Z}$ such that $u \in \mathcal{M}$ is regular whenever $u\left(\mathbb{C} P^{1}\right) \cap W_{\square} \neq \emptyset$. This can be done for example by perturbing $J$ on $W_{\square}$ suitably, see [5, 7]. With these generic choices the moduli space $\mathcal{M}$ is a smooth orientable manifold of dimension $4 d$.

(v) By the maximum principle in $\mathbb{C}^{d}$-direction as used in Lemma 3.2 (ii) and item (iii) above, the end of $\mathcal{M}$ is naturally diffeomorphic to the model $\left(\mathbb{C}^{d} \backslash B_{R}^{2 d}(0)\right) \times \mathbb{C} P^{d}$ via the correspondence between

$$
\left\{u \in \mathcal{M} \mid u\left(\mathbb{C} P^{1}\right) \cap\left\{\left(z_{1}, \mathbf{z}, w\right) \in \widehat{\operatorname{Cap}}|| \mathbf{z} \mid \geq R\right\} \neq \emptyset\right\}
$$

and

$$
\left\{u=(\mathrm{id}, \mathbf{z}, w)\left|\mathbf{z} \in \mathbb{C}^{d},\right| \mathbf{z} \mid \geq R, w \in \mathbb{C} P^{d}\right\} .
$$

We choose the orientation on $\mathcal{M}$ so that this correspondence preserves the orientations.

Lemma 3.4. If the evaluation map $\widehat{\mathrm{ev}}: \mathcal{M} \times \mathbb{C} P^{1} \rightarrow \hat{Z},(u, z) \mapsto u(z)$ is proper, then $W$ and $W_{\mathrm{st}}$ are diffeomorphic.

Proof. By definition of $\mathcal{M}$, each holomorphic sphere $u\left(\mathbb{C} P^{1}\right), u \in \mathcal{M}$, intersects $H_{1}$ at $\infty \in \mathbb{C} P^{1}$. This intersection is unique by positivity of intersections. Therefore, the evaluation map ev restricts to an evaluation map on $\mathcal{M} \times \mathbb{C}$ that takes values in $Z=\hat{Z} \backslash H_{1}$, i.e. $(u, z) \mapsto u(z)$ is a well-defined map ev: $\mathcal{M} \times \mathbb{C} \rightarrow Z$.

As $\widehat{\text { ev }}$ is proper, so is the restricted map ev, hence ev is of mapping degree 1 , see Remark 3.3 (v). Using the Umkehr-homomorphism based on Poincaré duality involving compactly supported cohomology, we see that ev is $H_{*}(., \mathbb{F})$-surjective for all fields $\mathbb{F}$. Surjectivity of ev in $\pi_{1}$ follows with a covering that corresponds to the index of the ev \#-image in $\pi_{1}(Z)$.

In view of Lemma 3.1 we consider the following commutative diagram:

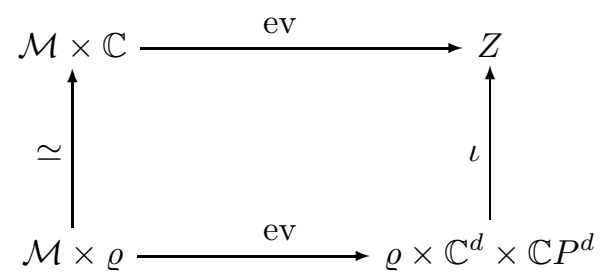

The induced diagram in $\pi_{1}$ and $H_{*}(., \mathbb{F})$ is commutative with upper ev $\#$ and $\mathrm{ev}_{*}$ surjective for all fields $\mathbb{F}$. Hence, $\iota_{\#}$ and $\iota_{*}$ are surjective. 
3.4. Properness of the evaluation map. We will investigate properness of the evaluation map. The results of this section are part of the proof of Theorem 1.1 For basics about holomorphic spheres we refer to McDuff-Salamon's book [7].

3.4.1. Gromov convergence. By the description of the end of $\mathcal{M}$ in Remark 3.3 (v) it suffices to consider sequences $\left\{u^{\nu}\right\}_{\nu}$ of holomorphic spheres $u^{\nu} \in \mathcal{M}$ whose images $u^{\nu}\left(\mathbb{C} P^{1}\right)$ are contained in

$$
W \cup\left\{\left(z_{1}, \mathbf{z}, w\right) \in \widehat{\operatorname{Cap}}|| \mathbf{z} \mid \leq R\right\} .
$$

Because the energy

$$
E\left(u^{\nu}\right)=\int_{\mathbb{C} P^{1}}\left(u^{\nu}\right)^{*} \hat{\Omega}=\pi r^{2}
$$

is uniformly bounded for all $\nu \in \mathbb{N}$, Gromov's compactness theorem applies: $u^{\nu}$ converges to a stable holomorphic sphere $\mathbf{u}$ with three marked points fixed. We denote by $u_{1}, \ldots, u_{N}, N \geq 1$, the non-constant components of $\mathbf{u}$. The convergence is in $C^{\infty}$ precisely if $N=1$. Therefore, the evaluation map êv is proper if $N=1$.

3.4.2. Intersections. The intersection number of $u^{\nu}$ and $H_{1}$ is $u^{\nu} \bullet H_{1}=1$ for all $\nu \in \mathbb{N}$. Furthermore a neighbourhood of $H_{1}$ is foliated by complex hypersurfaces of the form $z_{1} \times \mathbb{C}^{d} \times \mathbb{C} P^{d}$ with $\left|z_{1}\right|$ sufficiently large. Hence, we can assume that none of the $u_{j}\left(\mathbb{C} P^{1}\right), j=1, \ldots, N$, lies entirely in $H_{1}$, so that the intersection numbers of the bubbles $u_{j}$ and $H_{1}$ are defined, non-negative by positivity of intersections, i.e. $u_{j} \bullet H_{1} \geq 0$ for all $j=1, \ldots, N$, and sum up to $\sum_{j=1}^{N} u_{j} \bullet H_{1}=1$ according to Gromov convergence. Relabelling indices, if necessary, yields

$$
u_{1} \bullet H_{1}=1 \quad \text { and } \quad u_{j} \bullet H_{1}=0
$$

for all $j \geq 2$. By positivity of intersections and the maximum principle this implies that for each $j \geq 2$ we have the following alternative: either $u_{j}\left(\mathbb{C} P^{1}\right) \subset W_{\square}$ or $u_{j}=\left(\mathrm{pt}, \mathrm{pt}, u_{j}^{3}\right)$ for some holomorphic $u_{j}^{3}: \mathbb{C} P^{1} \rightarrow \mathbb{C} P^{d}$, see Lemma 3.2 (ii). In particular,

$$
u_{j}\left(\mathbb{C} P^{1}\right) \subset Z_{R}
$$

for all $j \geq 2$, where

$$
Z_{R}:=W \cup\left\{\left(z_{1}, \mathbf{z}, w\right) \in \operatorname{Cap}|| \mathbf{z} \mid \leq R\right\} .
$$

This will be used in the following section.

3.4.3. Implementing asphericity. We consider the homology classes $\left[u_{1}\right] \in H_{2} \hat{Z}$ and $\left[u_{j}\right] \in H_{2} Z_{R}, j \geq 2$, of the bubble spheres. Because the contact hypersurface $M=S T^{*} S^{2 d+1}$ is simply connected, we can decompose

$$
\left[u_{1}\right]=S_{1}+A_{1} \in H_{2} W \oplus H_{2} \widehat{\operatorname{Cap}}
$$

and

$$
\left[u_{j}\right]=S_{j}+A_{j} \in H_{2} W \oplus H_{2}\left(Z_{R} \backslash \operatorname{Int} W\right),
$$

for all $j \geq 2$, using a general position argument: The bubble spheres intersect $M$ along closed loops. These loops appear as the boundary of disc maps in $M$ for both orientations of the loops. Adding the disc maps to the disc parts of the bubble spheres corresponding to $W$ and its complements - respecting orientations - yields sphere maps that represent the $S_{j}$ and $A_{j}$ as desired. Since $(W, \omega)$ is symplectically 
aspherical, $\hat{\Omega}\left(S_{j}\right)=0$ for all $j=1, \ldots, N$. Consequently, using Stokes' theorem, we have

for all $j=1, \ldots, N$.

$$
0<E\left(u_{j}\right)=\hat{\Omega}\left(A_{j}\right)
$$

3.4.4. Enumeration. In order to compute the symplectic energy of the holomorphic spheres $u_{j}$ it suffices to compute $\hat{\Omega}\left(A_{j}\right)$ for the homology 2 -classes $A_{j}$ in $\widehat{\text { Cap }}$, see 3.4.3. Invoking the intersection pattern from 3.4.2 we find integers $\ell_{1}, \ldots, \ell_{N}$ such that

$$
A_{1}=\left[C_{1}\right]+\ell_{1}\left[C_{2}\right] \quad \text { and } \quad A_{j}=\ell_{j}\left[C_{2}\right],
$$

for all $j \geq 2$. Indeed, reading this as an equation in $H_{2} \widehat{\text { Cap }}$ this equation holds literally true if $d \geq 2$; if $d=1$, the equation holds up to possibly adding a multiple of the fibre class in $M=S T^{*} S^{3} \cong S^{3} \times S^{2}$. However, any representing fibre $S^{2}$ is disjoint from the complex hypersurface $H_{1}$ and does not contribute to the symplectic energy by local exactness of the symplectic form near $M$. For that reason we will not spell out the fibre class summands.

Positivity of energy from 3.4 .3 yields $r^{2}+\ell_{1}>0$ and $\ell_{j}>0$ for all $j \geq 2$. Furthermore, using $\hat{\Omega}\left(A_{j}\right)=\hat{\omega}\left(A_{j}\right)$ for all $j$,

$$
\pi r^{2}=E\left(u^{\nu}\right)=\sum_{j=1}^{N} \hat{\Omega}\left(A_{j}\right)=\pi r^{2}+\ell_{1} \pi+\ell_{2} \pi+\cdots+\ell_{N} \pi .
$$

It follows that $\ell_{1}+\cdots+\ell_{N}=0$. Therefore,

$$
N-1 \leq \ell_{2}+\cdots+\ell_{N}=-\ell_{1}<r^{2} .
$$

Taking $r \in(1, \sqrt{2}]$ in Section 3.2 the cases $N=1$ and $N=2$ remain. If $N=1$, then the evaluation map êv will be proper. If $N=2$, the above inequality turns into $1=N-1 \leq \ell_{2}=-\ell_{1}<2$ so that $\ell_{2}=-\ell_{1}=1$, i.e.

$$
A_{1}=\left[C_{1}\right]-\left[C_{2}\right] \quad \text { and } \quad A_{2}=\left[C_{2}\right] .
$$

The symplectic energies of the two bubble spheres satisfy

$$
E\left(u_{1}\right)=\left(r^{2}-1\right) \pi \quad \text { and } \quad E\left(u_{2}\right)=\pi .
$$

\section{Fittings}

Recall the cap construction from Section 3.1, that

$$
(Z, \Omega)=(W, \omega) \cup_{(M, \xi)}\left(\text { Cap, } \omega_{\text {Cap }}\right),
$$

and consider the complex hypersurface $H:=\mathbb{C}^{d+1} \times \mathbb{C} P^{d-1}$ in $\mathbb{C}^{d+1} \times \mathbb{C} P^{d}$.

4.1. Definition. A symplectic filling $(W, \omega)$ of $(M, \xi)=S T^{*} S^{2 d+1}$ is called a fitting if there exist a closed neighbourhood $U$ of $H$ in $\mathbb{C}^{d+1} \times \mathbb{C P}^{d}$ and an embedding $\varphi: U \rightarrow Z$ with $\left.\varphi\right|_{U \cap C a p}=$ id such that $\varphi_{*} J_{\text {st }}$ is tamed by $\Omega$ on $\varphi(U)$ and the homological intersection

$$
\varphi_{*}\left[H \cap D T^{*} S^{2 d+1}\right] \cdot S=0
$$

for all spherical classes $S \in H_{2} W$, i.e. for all classes in the image of the Hurewicz homomorphism. 
4.2. Proof of the Theorem 1.1. We show that $W$ and $W_{\text {st }}$ are diffeomorphic provided that $(W, \omega)$ is a symplectically aspherical fitting of $(M, \xi)$. We consider $(\hat{Z}, \hat{\Omega}, J)$ constructed in Section 3.3 and choose $r \in(1, \sqrt{2})$, see 3.4.4. The embedding $\varphi$ extends to an embedding (again denoted by $\varphi$ ) of a neighbourhood of $H_{2}=\mathbb{C} P^{1} \times \mathbb{C}^{d} \times \mathbb{C} P^{d-1}$ in $\mathbb{C} P^{1} \times \mathbb{C}^{d} \times \mathbb{C} P^{d}$ into $\hat{Z}$.

This extended embedding can be assumed to be holomorphic: The almost complex structure $J$ on $\hat{Z}$ equals the standard complex structure $J_{\text {st }}$ on $\hat{Z} \backslash W_{\square}$. We require $J$ to be $\varphi_{*} J_{\text {st }}$ in $\varphi(U)$ and perturb $J$ on $W_{\square} \backslash \varphi(U)$ to be generic for all holomorphic spheres that intersect the open set $W_{\square} \backslash \varphi(U)$ non-trivially.

In order to guarantee transversality of the moduli space $\mathcal{M}$ we need to verify regularity for all $u \in \mathcal{M}$ with $u\left(\mathbb{C} P^{1}\right) \subset \varphi(U)$. For those $u$, we obtain a holomorphic map

$$
\varphi^{-1} \circ u: \mathbb{C} P^{1} \longrightarrow \mathbb{C} P^{1} \times \mathbb{C}^{d} \times \mathbb{C} P^{d},
$$

which by the maximum principle can be written as

$$
\varphi^{-1} \circ u=(k, \mathrm{pt}, h)
$$

for holomorphic sphere maps $k$ into $\mathbb{C} P^{1}$ and $h$ into $\mathbb{C} P^{d}$. It suffices to show that $k$ is an automorphism of $\mathbb{C} P^{1}$ (and hence $k=$ id by the definition of $\mathcal{M}$ ) and that $h$ is constant: We compute symplectic energies of $\varphi^{-1} \circ u$ with respect to the possibly different symplectic forms $\varphi^{*} \hat{\Omega}$ and $\hat{\omega}$. The symplectic energy of $u$ equals

$$
\pi r^{2}=\hat{\Omega}([u])=\hat{\Omega}(A)
$$

by asphericity of $(\varphi(U) \cap W, \omega)$ and a splitting $[u]=S+A \in H_{2} W \oplus H_{2} \widehat{\text { Cap }}$, see 3.4.3. Because $\hat{\Omega}$ and $\hat{\omega}$ coincide on $\widehat{\mathrm{Cap}}$, this reads as $\pi r^{2}=\hat{\omega}(A)$. On the other hand, the symplectic energy of $\varphi^{-1} \circ u$ with respect to $\hat{\omega}$ equals

$$
\hat{\omega}\left(\left[\varphi^{-1} \circ u\right]\right)=\hat{\omega}(A),
$$

because $\left[\varphi^{-1} \circ u\right]=\left(\varphi^{-1}\right)_{*} S+A$ in $H_{2} W_{\text {st }} \oplus H_{2} \widehat{\operatorname{Cap}}$. This is because $\left.\varphi\right|_{U \cap \widehat{\text { Cap }}}=$ id and $W_{\mathrm{st}}=D T^{*} S^{2 d+1}$ has vanishing second homology. We see that

$$
\pi r^{2}=\hat{\omega}(A)=r^{2} \int_{\mathbb{C} P^{1}} k^{*} \omega_{\mathrm{FS}}+\int_{\mathbb{C} P^{1}} h^{*} \omega_{\mathrm{FS}}=a \pi r^{2}+b \pi
$$

for some non-negative integers $a, b$. It follows that

$$
r^{2}(1-a)=b .
$$

Since $1<r^{2}<2$ is not an integer $1-a$ must vanish, so that $a=1$ and $b=0$. This implies that the energy of $k$ is $\pi r^{2}$, i.e. $k$ is an automorphism on $\left(\mathbb{C} P^{1}, r^{2} \omega_{\mathrm{FS}}\right)$, and that $h$ has zero energy and, hence, must be constant. Therefore, $u$ is of the form $u=$ (id, pt,pt) and, in particular, $u$ is regular.

To finish the proof of Theorem 1.1 it remains to show properness of the evaluation map êv, see Lemma 3.4. In view of Section 3.4 we consider the case $N=2$ and $u_{1}$ as described. Suppose that $u_{1}\left(\mathbb{C} P^{1}\right)$ is not contained in the complex hypersurface $\varphi\left(H_{2}\right) \subset \hat{Z}$. By positivity of intersections, the intersection number

$$
\varphi\left(H_{2}\right) \bullet u_{1} \geq 0
$$

is defined and non-negative. On the other hand, $\varphi\left(H_{2}\right) \bullet u_{1}$ is equal to the homological intersection of $\left[\varphi\left(H_{2}\right)\right]=\varphi_{*}\left[H_{2}\right]$ with $\left[u_{1}\right]$. As in 3.4 .4 we use $A_{1}=\left[C_{1}\right]-\left[C_{2}\right]$ for the second summand of the decomposition $\left[u_{1}\right]=S_{1}+A_{1}$. If $d \geq 2$ such an equation is literally true; the second homology of $\widehat{\mathrm{Cap}}$ is the one of $\mathbb{C} P^{1} \times \mathbb{C}^{d} \times \mathbb{C} P^{d}$ 
by general position. Hence, $\left[u_{1}\right]=S_{1}+\left[C_{1}\right]-\left[C_{2}\right]$. The homological intersection condition in the definition of a fitting yields $\varphi_{*}\left[H_{2}\right] \cdot S_{1}=0$ as $S_{1}$ is spherical; the duality relations in Section 3.2 yield

$$
\varphi_{*}\left[H_{2}\right] \cdot\left[C_{1}\right]=0, \quad \varphi_{*}\left[H_{2}\right] \cdot\left[C_{2}\right]=1 .
$$

It follows that

$$
\left[\varphi\left(H_{2}\right)\right] \cdot\left[u_{1}\right]=-1
$$

contradicting positivity of intersections. If $d=1$, the equation $\left[u_{1}\right]=S_{1}+\left[C_{1}\right]-\left[C_{2}\right]$ holds up to adding a multiple of the fibre class in $S T^{*} S^{3} \cong S^{3} \times S^{2}$. Again by the definition of a fitting the fibre class contributes zero to the intersection with $\left[\varphi\left(H_{2}\right)\right]$. Therefore, we reach the same contradiction.

Consequently, $u_{1}\left(\mathbb{C} P^{1}\right) \subset \varphi\left(H_{2}\right)$. Analysing the energy $E\left(u_{1}\right)=\left(r^{2}-1\right) \pi$ in terms of $\varphi^{-1} \circ u_{1}=(k, \mathrm{pt}, h)$ as before we reach the equality

$$
\left(r^{2}-1\right) \pi=a^{\prime} \pi r^{2}+b^{\prime} \pi
$$

for non-negative integers $a^{\prime}, b^{\prime}$. In other words,

$$
r^{2}\left(1-a^{\prime}\right)=1+b^{\prime}
$$

with $1<r^{2}<2$. This is impossible. Therefore, $N=1$ and $\widehat{e v}$ is proper. By Lemma $3.4 W$ is diffeomorphic to $W_{\mathrm{st}}$. Q.E.D.

Acknowledgement. We thank Hansjörg Geiges for suggesting to use the term fitting, and his comments on the first draft of the manuscript.

\section{REFERENCES}

[1] M. Audin, F. Lalonde, L. Polterovich, Symplectic rigidity: Lagrangian submanifolds, in: Holomorphic curves in symplectic geometry, 271-321, Progr. Math., 117, Birkhäuser, Basel, 1994.

[2] K. Barth, H. Geiges, K. Zehmisch, The diffeomorphism type of symplectic fillings, to appear in J. Symplectic Geom.

[3] K. Barth, J. Schneider, K. Zehmisch, Symplectic dynamics of contact isotropic torus complements, Münster J. Math. 12 (2019), 31-48.

[4] H. Geiges, An introduction to contact topology, Cambridge Studies in Advanced Mathematics 109, Cambridge University Press, Cambridge (2008), xvi+440.

[5] H. Geiges, K. Zehmisch, Symplectic cobordisms and the strong Weinstein conjecture, Math. Proc. Cambridge Philos. Soc. 153 (2012), 261-279.

[6] D. McDuff, Symplectic manifolds with contact type boundaries, Invent. Math. 103 (1991), 651-671.

[7] D. McDuff, D. Salamon, J-holomorphic Curves and Symplectic Topology, Amer. Math. Soc. Colloq. Publ. 52, American Mathematical Society, Providence, RI (2004), xii+669.

[8] J. Milnor, Lectures on the h-Cobordism Theorem, Princeton University Press, Princeton, NJ (1965).

[9] S. Suhr, K. Zenmisch, Polyfolds, cobordisms, and the strong Weinstein conjecture, Adv. Math. 305 (2017), 1250-1267.

Mathematisches Institut, Justus-Liebig-Universität Giessen, Arndtstrasse 2, D-35392 Giessen, Germany

E-mail address: Myeonggi.Kwon@math.uni-giessen.de, Kai.Zehmisch@math.uni-giessen.de 